\title{
Optical System Design for High-Energy Particle Beam Diagnostics
}

\author{
Bingxin Yang \\ Argonne National Laboratory, 9700 South Cass Avenue, Argonne, IL 60439
}

\begin{abstract}
Radiation generated by high-energy particle beams is widely used to characterize the beam properties. While the wavelengths of radiation may vary from visible to x-rays, the physics underlying the engineering designs are similar. In this tutorial, we discuss the basic considerations for the optical system design in the context of beam instrumentation and the constraints applied by high-radiation environments. We cover commonly used optical diagnostics: fluorescence flags, visible and $\mathrm{x}$-ray synchrotron radiation imaging. Emphases will be on achieving desired resolution, accuracy, and reproducibility.
\end{abstract}

\section{INTRODUCTION}

The unique value of direct visualization of particle beams with optics and cameras was realized very early on in accelerator engineering. With the development of quantitative imaging tools, especially the ever better availability of CCD cameras and digitizers, optical imaging has become a vital part of particle beam diagnostics today. This tutorial is designed to introduce the field to starting young engineers and builds on several tutorials presented at past Beam Instrumentation Workshops [1-3]. The readers will also find it beneficial to be informed of progress in other fields that use quantitative imaging tools extensively, such as video microscopy, astronomy, and machine vision.

\section{Classification of Beam Imaging Techniques}

The goal of modern beam diagnostics is to transfer information carried by particle beams to computer memory, faithfully and efficiently. The transfer is always performed in several steps and uses several intermediate media. Figure 1 shows a typical optical diagnostic system: A conversion device that transfers the information from particle beam to radiation beam (light, x-ray, etc.), an optical system that forms the desired radiation pattern, a read-out device (camera) that converts the radiation intensity distribution into electrical signals, and a digitizer that converts the signal into discrete intensity maps to store in computer memory.

Any hardware component or media in the system can be and has been used to categorize optical diagnostics techniques, since changes in any component or information-carrying media could dramatically alter the system's performance and 
design criteria. For example, designs of electron and hadron diagnostics are very different since their length scale and beam dynamics are very different. In another example, efficient conversion devices and optics need to be used to take advantage of a fast detector (streak camera). This tutorial will be biased towards electron / positron diagnostics due to the author's own limited experience.

\begin{tabular}{|c|c|c|c|c|c|c|c|c|}
\hline $\begin{array}{c}\text { Particle } \\
\text { Beam }\end{array}$ & $\begin{array}{c}\text { Converter } \\
\text { Screens }\end{array}$ & Radiation & $\begin{array}{c}\text { Imaging } \\
\text { Optics }\end{array}$ & $\begin{array}{l}\text { Radiation } \\
\text { Pattern }\end{array}$ & $\begin{array}{c}\text { Readout } \\
\text { Device }\end{array}$ & $\begin{array}{l}\text { Video } \\
\text { Signal }\end{array}$ & \multirow{2}{*}{ Digitizer } & \multirow{2}{*}{$\begin{array}{l}\text { Computer } \\
\text { Memory }\end{array}$} \\
\hline $\begin{array}{l}\text { Electron } \\
\text { Proton / Ions }\end{array}$ & $\begin{array}{l}\text { Phosphor } \\
\text { OTR } \\
\text { Magnets }\end{array}$ & $\begin{array}{l}\text { Light } \\
\text { X-Ray }\end{array}$ & $\begin{array}{c}\overline{\text { Lens }} \\
\text { Mirror } \\
\text { Diffractive }\end{array}$ & $\begin{array}{l}\overline{\text { Direct }} \\
\text { Indirect }\end{array}$ & $\begin{array}{l}\text { CCD camera } \\
\text { Streak Cam } \\
\text { Many others }\end{array}$ & $\begin{array}{c}\text { Analog } \\
\text { Digital }\end{array}$ & & \\
\hline
\end{tabular}

FIGURE 1. Basic components of a beam imaging system: Rectangular boxes show informationcarrying media; arrow-shaped boxes show information transfer hardware.

An alternative but very important way to classify imaging techniques is by the type of point-spread function (PSF), which is the radiation intensity distribution formed with a single point source. When the PSF has a single, predominant peak, we call the optics a direct imaging system. The human brain readily understands images from such a system since the human eye images directly on retina. Here the transverse length scale of the PSF is characteristic of the image sharpness, and its rms length is often taken as the resolution. When the PSF is a multi-peaked pattern [4,5], often a type of interference pattern, we call the optics an indirect imaging system. This tutorial will deal mainly with direct imaging techniques.

The challenge facing the optics designer is best characterized by the system's angular resolution (viewed from the first optical element). This quantity will correctly take into account of wide variation of working distances (WD). Table 1 shows the typical dimensions of several common objects. While $1-\mu \mathrm{m}$ bacteria are "enormous" with a short WD microscope, a $2300-\mathrm{km}$ planet can be very small at an astronomical distance. As seen from the table, the angular sizes of particle beams fall in the same range of these common objects, depending on the working distance.

In the next section, we will first discuss screen-based (interceptive) beam imaging by going step-by-step through a working example, paying special attention to resolution issues and various contributing factors. In the following sections, we will discuss synchrotron-radiation-based (noninterceptive) imaging techniques, including visible light imaging and x-ray pinhole cameras.

TABLE 1. Angular Size of Several Sample Objects.

\begin{tabular}{cccccc}
\hline Object & Diameter & WD & Angular size & Optics & Comment \\
\hline Bacteria & $1 \mu \mathrm{m}$ & $0.63 \mathrm{~mm}$ & $1.6 \mathrm{mrad}$ & Microscope & Enormous \\
Human hair & $50 \mu \mathrm{m}$ & $25 \mathrm{~cm}$ & $200 \mu \mathrm{rad}$ & $\begin{array}{c}\text { Human eye } \\
\text { Reflective } \\
\text { telescope }\end{array}$ & Fair size \\
Pluto & $2300 \mathrm{~km}$ & $6 \times 10^{9} \mathrm{~km}$ & $0.4 \mu \mathrm{rad}$ & Tiny \\
Electron & \multirow{2}{*}{$30 \mu \mathrm{m}$} & $10 \mathrm{~cm}$ & $300 \mu \mathrm{rad}$ & $\begin{array}{c}\text { Light telescope } \\
\text { X-ray telescope }\end{array}$ & Fair size \\
beam & $10 \mathrm{~m}$ & $0.3 \mu \mathrm{rad}$ & Tiny \\
\hline
\end{tabular}




\section{SCREEN IMAGING SYSTEM DESIGN}

The basic type of imaging system for particle beams is based on converter screens (flags) using scintillation/fluorescence phenomena or relativistic effects (transition radiation, Cherenkov radiation, etc.). With the appropriate choice of scintillator material, the screen can also be used for x-ray beam imaging. In this section, we will explore various aspects of design and modeling by working at an actual system [6].

The Advanced Photon Source (APS) free-electron laser system uses low-emittance electron beams. In the bunch compressor region, where the beam is strongly focused, the smallest beam is around $50 \mu \mathrm{m}$ in rms radius, and the bunch charge is about 0.2 $\mathrm{nC}$. On our wish list, we have asked for a $17-\mu \mathrm{m}$ rms resolution, and $0.2-\mathrm{nC}$ charge sensitivity. A model study also showed that 5\% accuracy in beam-size measurements was needed to support the studies of the coherent synchrotron radiation effect [7]. To increase the charge sensitivity, we also asked for the highest acceptance solid angle and the largest of field of view (FOV) the vacuum enclosure allows.

\section{Technical Specification and Consistency}

Before turning the wish list into technical specifications, we perform several consistency checks. While some of them are based on basic optical principles, others merely reflect hardware limitations.

\section{(1) FOV-to-resolution ratio}

Any digitized image contains a finite number of picture elements (pixels), which imposes restrictions on the size of the FOV for a given resolution. The APS uses standard RS-170 video systems. While the horizontal pixel number can vary from 400 to 900 for different camera / digitizer combinations, the maximum number of vertical lines is 483 . Using a criterion of a minimum two pixels per resolution element, we have the maximum field of view given by

$$
\frac{\text { FOV }}{\text { Resolution }} \leq \frac{\text { max pixel \# }}{2}=240 .
$$

This yields a 4-mm FOV in the vertical direction. To overcome this limitation, one could use CCD cameras with more pixels; a zoom lens to change magnification; or two cameras, one set at high resolution and one set at full field of view, sharing the light with a beam splitter or switching mirror. Once the CCD is chosen and the FOV is decided upon, the optical system magnification is then given by the ratio of pixel sizes at the image and object planes.

\section{(2) Phase-space acceptance limit}

Let us consider a simple imaging system made of two lenses, with the object and CCD chip placed at the respective focal point (Fig. 2). The magnification of the system $(M)$ is given by the ratio of the object height $(h)$ and image height $\left(h^{\prime}\right), M=h^{\prime} / h=S^{\prime} / S$. Since the FOV in the image space is limited by the effective size of the CCD chip 
$l_{\mathrm{CCD}}$, and the $\mathrm{F}$ number of the lenses are also limited practically, we obtain the phasespace limitation for the entire imaging system,

$$
F O V \cdot \text { Total collection angle }=\frac{2 h D}{S}=\frac{2 h^{\prime} D}{S^{\prime}} \leq \min \left\{\frac{l_{C D D}}{M F_{1}}, \frac{l_{C D D}}{F_{2}}\right\},
$$

where $F_{1}=D / S$ and $F_{2}=D / S^{\prime}$ are the $F$ numbers of the lenses. Since the phase-space volume remains a constant through out an optical system, this limitation is fairly general in nature. It means that a large field of view and a large collection angle cannot be obtained at the same time with a single imaging system, and a compromise needs to be made. To increase total phase-space acceptance, one can use a large lens (small $F$ number), or if the system is demagnifying $(M<1)$, use a large CCD chip.

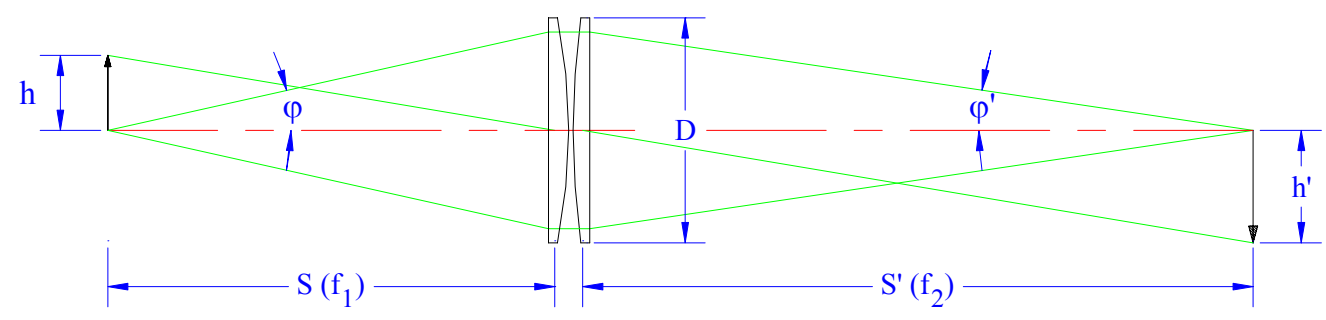

FIGURE 2. A simple imaging system using two infinity-conjugate lenses. The diameters of the lenses are $D$, and their focal lengths are $f_{1}$ and $f_{2}$. The object distance $S=f_{1}$ and the image distance $S^{\prime}=f_{2}$.

\section{(3) Working distance and shielding requirements}

Unless the radiation generated by the particle beam is low, the CCD cameras need to be shielded to prevent radiation damage (snowy pictures). A simple but effective way is to make one or more bends in the optical path so that high- $\mathrm{Z}$ materials (W or $\mathrm{Pb}$ ) can be placed to block the radiation generated at the screen from reaching the camera. For high-energy particle beams, radiation shower originating from upstream points of the accelerator should also be considered.

To perform radiation ray-tracing, all low- $Z$ materials must be ignored. Often it is convenient to trace backwards from the camera (acceptance zones). The additional bends and shielding geometry lengthens the optical paths and reduces the $F$ numbers of the suitable lenses, further restricting the phase-space acceptance volume.

After applying the above three constraints, we reached a set of realistic design specifications for the APS flag system (Table 2). The phase-space acceptance is based on a CCD chip $6.4 \mathrm{~mm} \times 4.8 \mathrm{~mm}$ in size.

TABLE 2. Specifications for the APS Bunch Compressor Flag.

\begin{tabular}{ccc}
\hline Camera / Optics & High Resolution & Low Resolution \\
\hline Resolution & $17 \mu \mathrm{m}$ & $50 \mu \mathrm{m}$ \\
Working distance & $125 \mathrm{~mm}$ & $200 \mathrm{~mm}$ \\
Field of view (FOV) & $6.4 \times 4.8 \mathrm{~mm}^{2}$ & $20 \times 15 \mathrm{~mm}^{2}$ \\
Light collection angle & 0.2 radian & 0.12 radian \\
Phase-space acceptance & $1.3 \times 1(\mathrm{~mm}-\mathrm{rad})^{2}$ & $2.4 \times 1.8(\mathrm{~mm}-\mathrm{rad})^{2}$ \\
\hline
\end{tabular}




\section{Selection of Converter Screen and Geometry}

Converter screens are available in various types and forms, and most of them are based on ionizing-particle-induced scintillation (Fig. 3). For relativistic particles, an optical transition radiation (OTR) screen is also an option.

\section{(1) Powdered phosphor $[1,2]$}

A phosphor screen is formed by adhering phosphor powder to a substrate. The scintillation light reflects multiple times before escaping the powder particle, thus lighting up the entire grain. In a single layer, the resolution is limited by the average size of the grains, typically several to several tens of micrometers. These screens are economical and versatile due to their simple fabrication process. Phosphors with different spectra are available to fulfill the needs of optical system requirements. Phosphors with decay time as short as 45 ps are available for dynamic studies [1].

\section{(2) Ceramic phosphors}

Ceramic phosphor screen can be considered as sintered screen with fine grains of phosphor powder. The sizes and thicknesses of the screens are usually made to order. They are self-supporting and can be machined only with special tools. Since the grains are coupled closely optically, the spatial resolution of the screens is normally given by the size of several grains, usually in the range of 100 micrometers or more. Not many choices are available in this category. Commonly used screen materials include Chromax ( $\mathrm{Cr}$ doped $\left.\mathrm{Al}_{2} \mathrm{O}_{3}\right)$ and YAG:Ce.
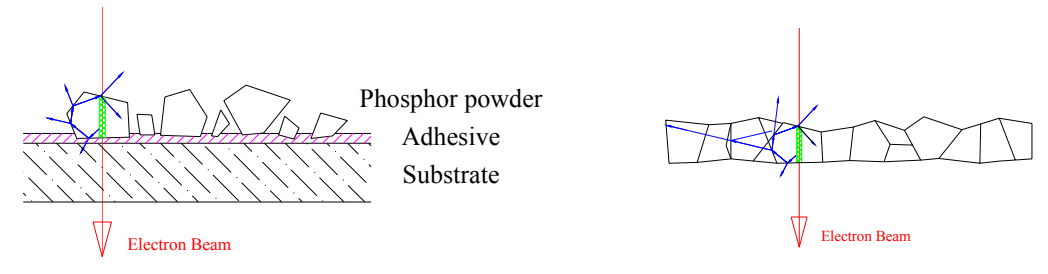

FIGURE 3. (Left) Schematics of a powdered phosphor screen. (Right) Ceramic phosphor screen.

\section{(3) Clear plastic scintillators}

Plastic scintillators have good efficiencies and short decay times, but their use in beam imaging is very rare, probably due to their low radiation damage threshold (molecular instead of ionic crystals) or poor vacuum compatibility.

\section{(4) Translucent inorganic single-crystal scintillators}

Cerium-doped yttrium aluminum garnet (YAG:Ce) was proposed as an image converter screen for electron microscopy by R. Autrata et al. in 1983 [8]. However, its actual application had been very limited due to the crystal's high cost. In the ensuing decade, large quantities of YAG crystals were produced for the laser industry and its price has dropped significantly. Interest in the translucent inorganic scintillators has been revived recently $[9,10]$ and other crystals (YAP:Ce, LSO:Ce) have also been tested. 
Compared with phosphor screens, the inorganic scintillators have significant advantages. They are economical, compatible with ultra-high vacuum, efficient in light conversion, highly resistant to beam damage, with narrow emission spectrum (reducing chromatic aberration), and most importantly, with good spatial resolution. In the case of YAG crystals, reported resolution ranges from $40 \mu \mathrm{m}$ for high charge density beam, to $10 \mu \mathrm{m}$ for low density ones, and even $1 \mu \mathrm{m}$ or less for specially fabricated YAG crystals with only a several- $\mu$ m-thick Ce-doped layer. A saturationlike blurring starting at a charge density of $6-20 \mathrm{nC} / \mathrm{mm}^{2} /$ bunch appears to be a significant limitation for the spatial resolution of YAG and other crystals $[11,12]$.

\section{(5) Optical transition radiation (OTR) screens [13]}

When charged particles traverse the interface between different indices of diffraction, photons are emitted. When a metal foil is used, OTR is emitted in the specular direction from the front surface where electrons enter the metal (reflective OTR) and also in the forward direction from the back surface where electrons exit the metal (forward OTR). The radiation is radially polarized, and its angular distribution is concentrated in a cone with a radius of $1 / \gamma$ and a dark center:

$$
\frac{d^{2} N}{d \omega d \Omega} \propto \frac{\theta_{x}{ }^{2}+\theta_{y}{ }^{2}}{\left(\gamma^{-2}+\theta_{x}{ }^{2}+\theta_{y}{ }^{2}\right)^{2}},
$$

where $\theta_{\mathrm{x}}$ and $\theta_{\mathrm{y}}$ are angles of observation from the specular / forward direction for the two types of OTR, respectively. The photon spectrum spans a wide range, and the angle-integrated photon flux in the frequency region of $\left[\omega_{1}, \omega_{2}\right]$ is given by

$$
n\left(\omega_{1}, \omega_{2}\right)=\frac{2 \alpha}{\pi} \ln \gamma \cdot \ln \frac{\omega_{2}}{\omega_{1}} .
$$

For $200-\mathrm{MeV}$ electrons $(\gamma \approx 400)$, about one visible photon is produced for every 60 electrons. This can be compared to $10^{4} \sim 10^{5}$ photons generated in YAG by the same number of electrons. However, the scintillation photons are emitted in all $4 \pi$ solid angles, and a practical imaging system could only capture $<1 \%$ of the light, while the same system would capture a large percentage of OTR photons.

For this APS flag project, we will use one 0.1-mm-thick YAG scintillator for the converter screen at low charge intensity and one OTR screen (a 45 degree mounted metal mirror) at high intensity.

\section{Error Management}

Emittance measurements with 10\% accuracy are routinely performed with basic hardware, except when the statistics are really poor. For measurements with 5\% accuracy or better, all sources of error need to be considered and each one carefully managed (Table 3). Defects of screens (dopant concentration variation, etc.) and optics (scratches, oxidation, or contamination) are difficult to control or model. The tolerance budgets assigned to these sources are somewhat arbitrary, but their total effect can be measured experimentally. The statistical fluctuation of photons collected by the 
camera is expected to be a major source of error since each bunch contains only 0.2$\mathrm{nC}$ of charge, or $1.2 \times 10^{9}$ electrons. For an OTR screen, this corresponds $\sim 2 \times 10^{7}$ photons spreading out in the entire image. Assuming the accelerator is stable, successive single-bunch measurements will show the statistical fluctuation of the measured beam size.

In the next two subsections, we will discuss the modeling of the resolution and calibration and quality assurance issues.

TABLE 3. Tolerance Budget for the APS Bunch Compressor Flag.

\begin{tabular}{ccc}
\hline Source of Error & Size Tolerance & Emittance Tolerance \\
\hline Screen defects & $1.0 \%$ & $1.4 \%$ \\
Optics defects & $1.0 \%$ & $1.4 \%$ \\
Resolution & $2.0 \%$ & $2.8 \%$ \\
Calibration & $1.4 \%$ & $2.0 \%$ \\
Statistics & $2.0 \%$ & $2.8 \%$ \\
Total & $3.5 \%$ & $5 \%$ \\
\hline
\end{tabular}

\section{Resolution Analysis}

Resolution of optical instruments are defined differently in different fields. Microscopists like to use the Rayleigh criteria, while broadcasting video uses line pairs per screen. In beam physics, rms resolution is widely used; for Gaussian PSF, this definition results in a simple quadrature relationship between the measured source size $\sigma$, and the true source size $\sigma_{0}$,

$$
\sigma^{2}=\sigma_{0}^{2}+\sigma_{R}^{2}
$$

where $\sigma_{\mathrm{R}}$ is the rms width of the PSF and is called the instrument's resolution. This definition encounters two major difficulties in practice: First, a true rms calculation is prone to background noise or drift. Second, for non-Gaussian PSF, the simple quadrature relation breaks down, and a fit to the exact convolution is often used to determine the actual source size. As an example to illustrate the mathematical difficulty, the one-dimensional diffraction PSF, the sinc function, has an infinite rms width. To avoid the above technical difficulties, we propose to define an effective Gaussian resolution based on the quadrature relation.

\section{Definition of Effective Gaussian Resolution}

Assume that we are measuring a Gaussian source,

$$
g_{0}(x)=\frac{A_{0}}{\sqrt{2 \pi} \sigma_{0}} e^{-x^{2} / 2 \sigma_{0}^{2}}
$$

with an instrument with a point spread function $h(x)$. The resultant intensity distribution is then given by

$$
f(x)=\int_{-\infty}^{\infty} g_{0}\left(x-x^{\prime}\right) h\left(x^{\prime}\right) d x^{\prime}, a \leq x \leq b .
$$


After fitting $f(x)$ with a Gaussian function $g(x)=\frac{A}{\sqrt{2 \pi} \sigma} e^{-x^{2} / 2 \sigma^{2}}$, i.e., with minimizing

$$
\chi(A, \sigma) \equiv \int_{a}^{b}[f(x)-g(x)]^{2} d x,
$$

we obtain the effective Gaussian height $A$ and width $\sigma$. We now define the effective (Gaussian) resolution of the instrument as

$$
\sigma_{R}=\sqrt{\sigma^{2}-\sigma_{0}^{2}}
$$

When the PSF is an exact Gaussian function, this is identical with the rms definition. However, if the PSF is not Gaussian, the resolution could be a function of source size!

\section{Image Formation and Diffraction Point Spread Function [14]}

In geometrical optics, a perfect imaging system converts a bundle of rays radiating from a single point (source) to a bundle converging to a single point (image). By the same token, a perfect imaging system in wave optics converts an outgoing single spherical wavefront centered at the source point to another converging single spherical wavefront centered at the image point $O$, as shown in the coordinate system in Fig. 4.

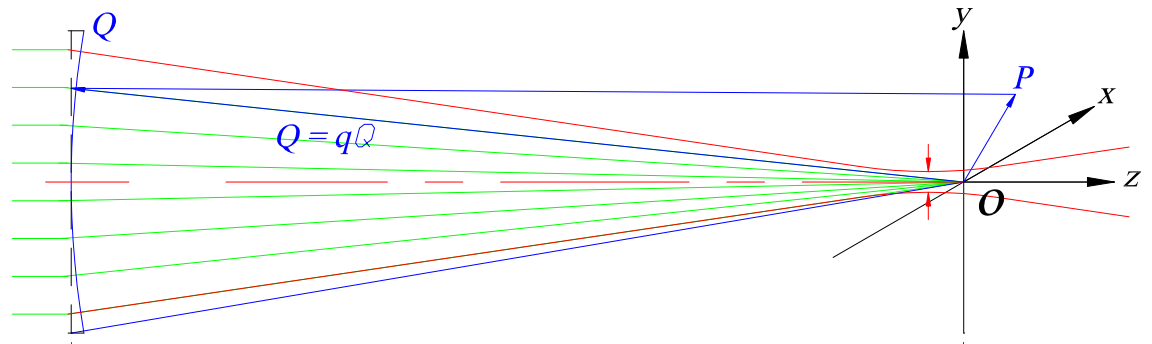

FIGURE 4. Coordinate system near the classical focus (image point) $O$. The $z$-axis is chosen to be the optical axis, $y$ is vertical, and $\mathrm{x}$ is defined by the right-hand rule. Point $Q$ is located in the exit plane of the optical system.

Using the rays passing through the classical focus as the reference path, the path difference for any line connecting a point $Q$ on the exit surface and a point $P$ near the focus can be obtained. Applying Huygens Principle, the light intensity distribution (PSF) near the classical focus can be expressed by a Helmholtz diffraction integral,

$$
\begin{aligned}
& h_{\text {diff }}(x, y, z)=\left|A_{\text {diff }}(x, y, z)\right|^{2} \text {, and } \\
& \qquad A_{\text {diff }}(x, y, z)=\int A(\mathbf{q}) e^{-i \mathbf{q} \mathbf{P} / \lambda} d \Omega=\int A(\mathbf{q}) e^{-\frac{i}{\lambda}\left(x \cos \phi_{x}+y \cos \phi_{y}+z \cos \phi_{z}\right)} d \Omega,
\end{aligned}
$$

where the complex aperture function $A(\boldsymbol{q})$ is the light amplitude in the $\boldsymbol{q}\left(\cos \phi_{x}, \cos \phi_{y}\right.$, $\cos \phi_{z}$ ) direction far away from the focus. This integral has been well studied. Several well-known properties are listed here:

(1) The minimum beam waist is not located at the geometrical focus but between the focus and the optics. The difference is not significant in most cases unless the light cone angle falls in mrad-range or below. 
(2) For uniform illumination, the PSF at the focal plane has a predominant peak with a length scale $\lambda / \theta_{\max }$, and many side peaks (diffraction pattern).

(3) A fairly general form of the Uncertainty Principle may be proved

$$
\sqrt{\left\langle\Delta x^{2}\right\rangle} \sqrt{\left\langle\Delta \theta_{x}^{2}\right\rangle} \leq \frac{\lambda}{2}=\frac{\lambda}{4 \pi}
$$

relating the rms deviation of the transverse coordinates and momentum. The minimum size of the beam given by this expression is often referred to as the diffraction limit.

As an example, we use the Uncertainty Principle to estimate the resolution of OTR imaging with a cone angle of $\theta_{\max }$. Using the angular distribution of Eq. (4), it is straightforward to derive

$$
\sqrt{\left\langle\theta_{x}^{2}\right\rangle} \approx \frac{\theta_{\max }}{\sqrt{\left(8 \ln \gamma \theta_{\max }-4\right) / 3}} .
$$

Other than a very slowly varying denominator, this expression is similar to the case of uniform illumination, $\sqrt{\left\langle\theta_{x}^{2}\right\rangle} \square \theta_{\max } / \sqrt{2}$, hence we conclude that the diffraction limit of the OTR imaging is not very different from the case of uniform illumination. This estimate is consistent with the exact PSF calculation using Eq. (4) as the aperture function in Eq. (11) [15].

When the aperture is illuminated uniformly and is rectangular in shape, the PSF is a product of two sinc functions, $h_{\text {diff }}(x, y)=h_{\text {diff }}(x) \cdot h_{\text {diff }}(y)$, with each one given by

$$
h_{\text {diff }}(x)=\left[\sin \left(\frac{x \theta_{\max }}{\lambda}\right) /\left(\frac{x \theta_{\max }}{\lambda}\right)\right]^{2} \text {. }
$$

Its effective resolution can be modeled with numerical calculation (Fig. 5). The results in the region of practical interest can be parameterized with a simple formula,

$$
\sigma_{\text {Res }, \text { iff }}=0.36 \cdot l_{d}\left\{1+2\left(\frac{\sigma_{0}}{l_{d}}\right)^{2}\right\}^{1 / 4}, \quad\left(l_{d}=\frac{\lambda}{2 \theta_{\max }}\right) .
$$

For a point source, the Gaussian fit only uses the main peak (Fig. 5). For an extended source, the convolution incorporates sidelobes into the main peak, and the effective resolution grows as source size increases.

SINC FUNCTION LENGTH SCALE

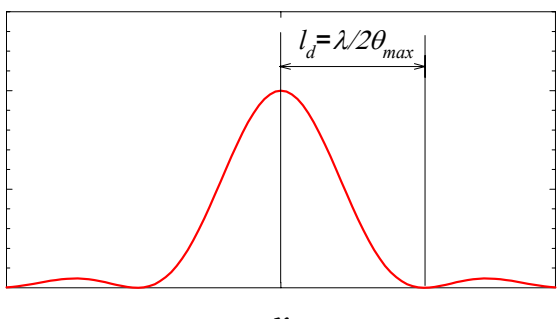

$x$

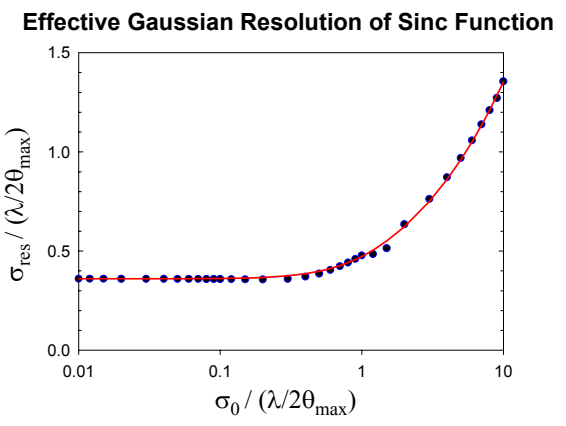

FIGURE 5. (Left) One-dimensional diffraction PSF, a sinc function. (Right) The effective Gaussian resolution of the diffraction PSF. 


\section{Charge Binning and Spilling in the CCD Pixels}

All CCD camera elements have finite sizes. The charges generated over a finite area are integrated and presented as one point (binning). When the light is shining on one CCD pixel, its neighbor may also get charged due to spilled charge or scattered radiation. These effects can be modeled numerically and the results are shown in Fig. 6 . The result can be roughly summarized as

$$
\sigma_{R, p i x} \approx 0.3+\text { spill_fraction }
$$

In the model used here, the spill_fraction is the fraction of charge spilled to the nearest neighbors, evenly divided between its left and its right.

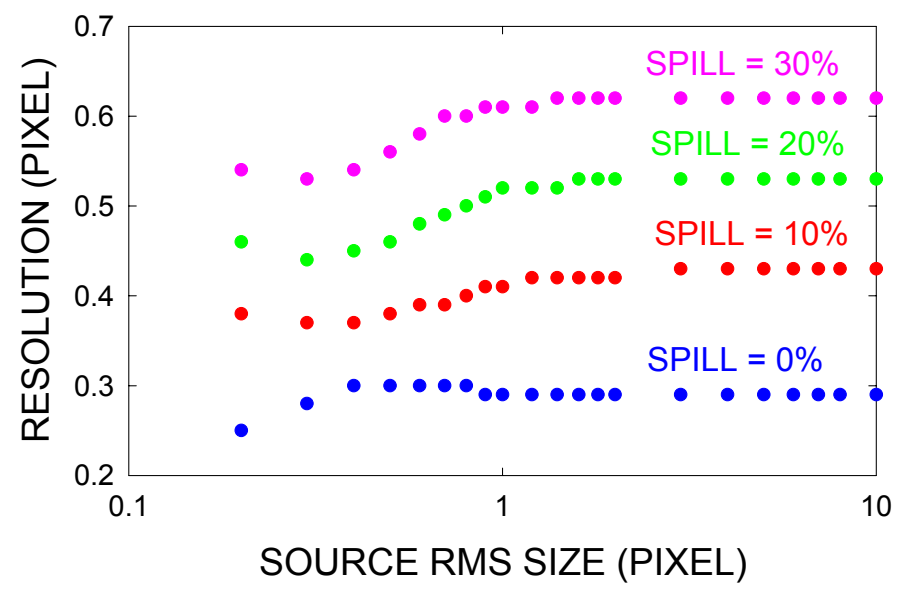

FIGURE 6. Effective Gaussian resolution due to complete binning. From bottom to top, $0 \%, 10 \%$, $20 \%$, or $30 \%$ charge of every pixel is spilled to its nearest neighbors, evenly divided to its left and right.

\section{Effective Resolution from Defocus}

If we shift the source point away from the ideal object plane by a distance $z$, the light cone eventually collected by the optics would illuminate a disc uniformly at the ideal object plane (Fig. 7). If the system were perfect in the sense of geometrical optics, this disc would be mapped faithfully to the camera. The PSF is thus a uniform disc with a radius $\left|\mathrm{z} \theta_{\max }\right|$. After integrating over y we obtain a one-dimensional PSF

$$
h_{\text {defocus }}(x)=\frac{2 \sqrt{z^{2} \theta_{\max }{ }^{2}-x^{2}}}{\pi z^{2} \theta_{\max }{ }^{2}}, \quad\left(x^{2} \leq z^{2} \theta_{\max }{ }^{2}\right) \text {. }
$$

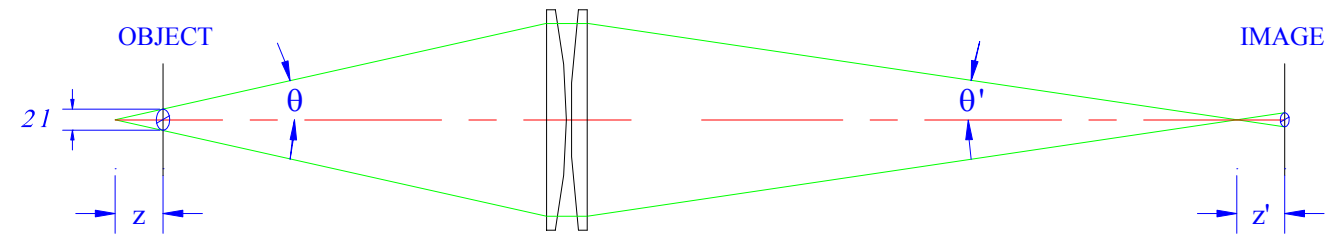

FIGURE 7. Diffusion of light due to defocus: the actual source is located away from the ideal object place by a distance $z$. 
The effective resolution can be modeled numerically (Fig. 8). For all practical purposes when defocus is not the dominant factor, we have,

$$
\sigma_{R, \text { defocus }}(x) \approx 0.5\left|z \theta_{\max }\right| \text {. }
$$

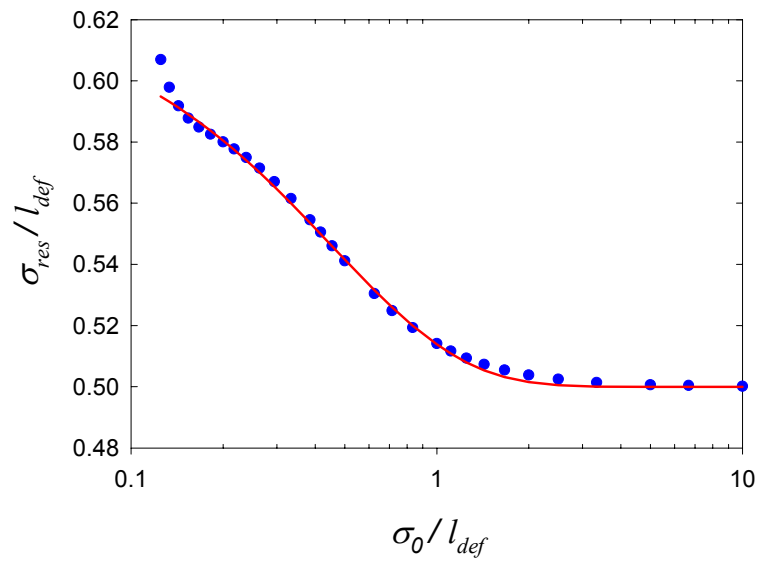

FIGURE 8. Gaussian resolution due to defocusing. Length unit $l_{d e f}=\left|z \theta_{\max }\right|$.

\section{Effective Resolution from Depth of Source}

At normal incidence, translucent YAG screens can be modeled as uniform sources along the optical axis, extending from $z_{1}$ to $z_{2}$, with $z=0$ at the ideal object plane. The integration of Eq. (15) along the $z$-axis gives a PSF,

$$
h_{\text {depth }}\left(x ; z_{1}, z_{2}\right)=\int_{z_{1}}^{z_{2}} \frac{2 \sqrt{z^{2} \theta_{\max }^{2}-x^{2}}}{\pi z^{2} \theta_{\text {max }}^{2}\left|z_{2}-z_{1}\right|} d z=\frac{H\left(x ; z_{2}\right)-H\left(x ; z_{1}\right)}{\left|z_{2}-z_{1}\right|} \quad\left(x \leq\left|z \theta_{\max }\right|\right) \text {, }
$$

where

$$
H(x ; z)=\left\{\begin{array}{ll}
\frac{2}{\pi \theta_{\max }}\left[\operatorname{ch}^{-1}\left(\frac{z \theta_{\max }}{x}\right)-\frac{\sqrt{z^{2} \theta_{\max }^{2}-x^{2}}}{z \theta_{\max }}\right] & \left(|x| \leq z \theta_{\max }\right) \\
0 & \left(|x|>z \theta_{\max }\right)
\end{array} .\right.
$$

Its effective resolution can be numerically modeled and again parameterized approximately as

$$
\sigma_{R E S}^{2}\left(\rho_{1}, \rho_{2}\right) \approx \frac{\left(\rho_{1}+\rho_{2}\right)^{2}}{16}+\frac{\left(\rho_{1}-\rho_{2}\right)^{2}}{48}+\frac{\rho_{1} \rho_{2}\left(\rho_{1}+\rho_{2}\right)^{2}}{1024 \cdot \sigma_{0}^{2}},
$$

where $\rho_{i}=\frac{z_{i} \theta_{\max }}{n}$, and $n$ is the index of refraction of the scintillator. As expected, minimum blurring occurs when the center of the YAG is at the ideal focal plane, with the minimum resolution given by

$$
\sigma_{R E S \text { min }}\left(\rho_{1}, \rho_{2}\right)=\frac{\left|\rho_{1}-\rho_{2}\right|}{4 \sqrt{3}}=\frac{\left|z_{1}-z_{2}\right| \theta_{\max }}{4 n \sqrt{3}} .
$$


For a source located away from the optical axis, the discs from different parts of the line source do not overlap concentrically. It results in a comet-like PSF.

Multiple reflections from front and back surfaces could increase the effective source depth. When the front surface is coated with reflective metal, it effectively doubles the source depth. Otherwise, unless the scintillator crystal or CCD camera is saturated, the reflected light adds only minor tails to the PSF since its intensity is reduced by $1 / n$ by each reflection.

\section{Contribution of Geometrical Aberrations}

In geometrical optics, an ideal lens converts a bundle of rays from a source point to a bundle converging to a single image point. For a realistic optic however, the converging rays do not always pass through the image point. The deviation is the aberration, and the density distribution of these rays at the image plane forms the geometric PSF.

Detailed discussions of geometric aberrations can be found in standard optics textbooks. For beam diagnostics, two types of aberrations are important: spherical and chromatic aberrations. The former comes from the over-focusing of large-angle rays away from the optics axis, and results in focal lengths changing with pupil size. It can be reduced by using small apertures and adjusting the focus after every change of aperture. The latter comes from wavelength dependence of the refractive indices of lens materials, and results in defocusing-like blurring of the PSF. The effect can be reduced with the use of an achromatic lens, a scintillator with spectrum matching the optics, or a bandpass filter to limit the spectral width. It can be totally eliminated by the use of mirror optics.

\section{Ray-Tracing Analysis}

A ray-tracing program is not only indispensable for the analysis of the geometric aberrations, but also useful for other types of PSF analyses discussed above. Figure 9 shows a sample screen output from one ray-tracing program, ZEMAX [16]. It gives information about ray offsets from the ideal image point due to their angular and wavelength offset. Moving the source point in the transverse direction, the elongation of a spot for an off-axis source point shows the distortion of the PSF across the field of view. Moving the source point in the longitudinal direction, the change of the spot diagram shows the effect of defocus. Table 4 shows such an effort for the APS chicane flag. From the table, we make the following observations, (1) for monochromatic light, the diffraction-limited resolution dominates at the focal plane; (2) for the narrow-band optics (corresponding to the spectrum of YAG scintillation), the resolution is dominated by the chromatic aberration; (3) for the broadband optics (corresponding to the OTR spectrum), the resolution further worsens by about a factor of two; and (4) when the object is $200 \mu \mathrm{m}$ out of focus, the defocusing starts to dominate the total resolution. We also conclude that the resolution for this design is within the budget given in Table 3 . 


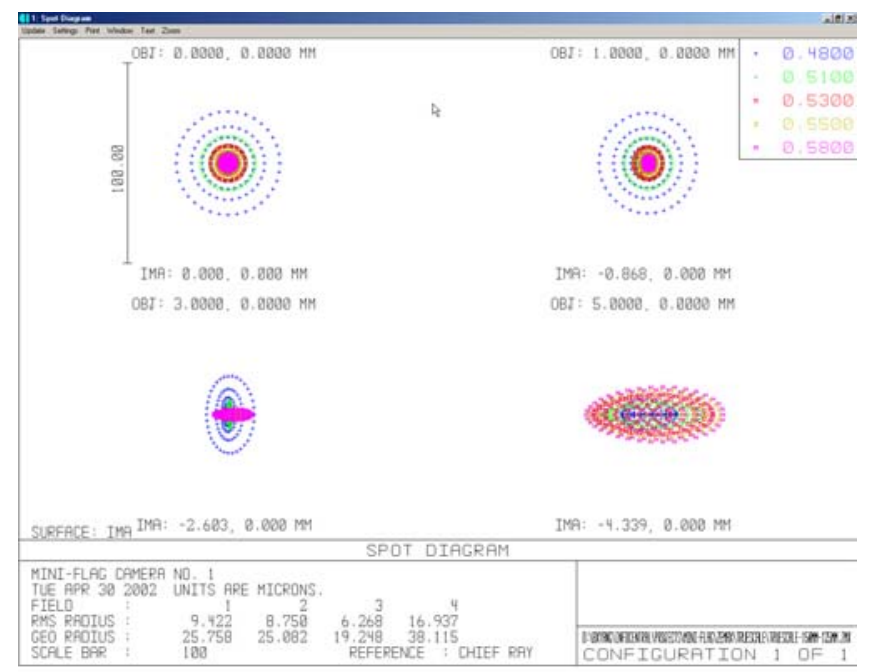

FIGURE 9. A "spot-diagram" from ZEMAX, a ray-tracing program, shows the distribution of rays at the focal plane for different wavelengths, and for on-axis and off-axis source points.

TABLE 4. Rms Radii of the PSF Calculated with ZEMAX.

\begin{tabular}{cccc}
\hline Configuration & Monochromatic & Narrow band & Broadband \\
\hline Wavelength $(\mathrm{nm})$ & 550 & $530 \pm 50$ & $450-700$ \\
Diffraction peak & $1.5 \mu \mathrm{m}$ & $1.5 \mu \mathrm{m}$ & $1.7 \mu \mathrm{m}$ \\
Vacuum path alone & $1.60 \mu \mathrm{m}$ & $5.2 \mu \mathrm{m}$ & $9.0 \mu \mathrm{m}$ \\
+3.2 mm window & $1.61 \mu \mathrm{m}$ & $5.4 \mu \mathrm{m}$ & $9.1 \mu \mathrm{m}$ \\
+ Beam splitter \& $90^{\circ}$ prism & $1.8 \mu \mathrm{m}$ & $6.5 \mu \mathrm{m}$ & $10.3 \mu \mathrm{m}$ \\
Defocus $+200 \mu \mathrm{m}$ & $10.1 \mu \mathrm{m}$ & $13.4 \mu \mathrm{m}$ & $15.1 \mu \mathrm{m}$ \\
Defocus $-200 \mu \mathrm{m}$ & $10.9 \mu \mathrm{m}$ & $10.4 \mu \mathrm{m}$ & $14.1 \mu \mathrm{m}$ \\
\hline
\end{tabular}

We wish to make two notes at the end of this resolution analysis. First, strictly speaking, the quadrature sum rule no longer holds for more than two terms. But in practice, the system resolution is usually dominated by one or two components. Variations of other terms are not important in the overall picture. Here one can lump them with the source size before the analysis of the dominant resolution term.

Secondly, the full wave-optic treatment of light propagation through an instrument, a dream of all optics designers, has recently become available in commercial raytracing products. When it matures, the analytical expressions of resolution will be of only semiquantitative value, just like the geometric ray-tracing programs have already made the analytical expressions of aberration semiquantitative tools.

\section{Calibration}

Many calibration techniques are in use today, fitting a wide range of budgets and needs for accuracy: (1) known machined features in the FOV, a hole, a slot, or a set of pinholes; (2) grid patterns, especially those formed by small holes / bright spots; or (3) scanning pinholes moved by computer-controlled stages. The key to reliable calibration appears to employ features that the computer recognizes, i.e., imaging software can easily analyze dimensions of the features. For example, using matrices of 
small holes as calibration targets, their centroid can be used for camera scale calibration while their width can be used for resolution measurements.

The most accurate characterization technique is the use of a back-lit, scanning pinhole moved by computer controlled linear stages [6]. Scanning the pinhole in the longitudinal direction can locate the focal point and determine defocusing properties. Scanning the pinhole in the transverse direction can calibrate the camera scales and measure the resolution change across the field of view (Fig. 10). Adding variations of the wavelength, the 4-dimensional scan can be used to fully characterize the imaging system. Furthermore, the same calibration procedures can be automated and performed many times for consistency checks. Our experience shows that only the scanning pinhole technique has the reproducibility to meet the requirements in Table 3.
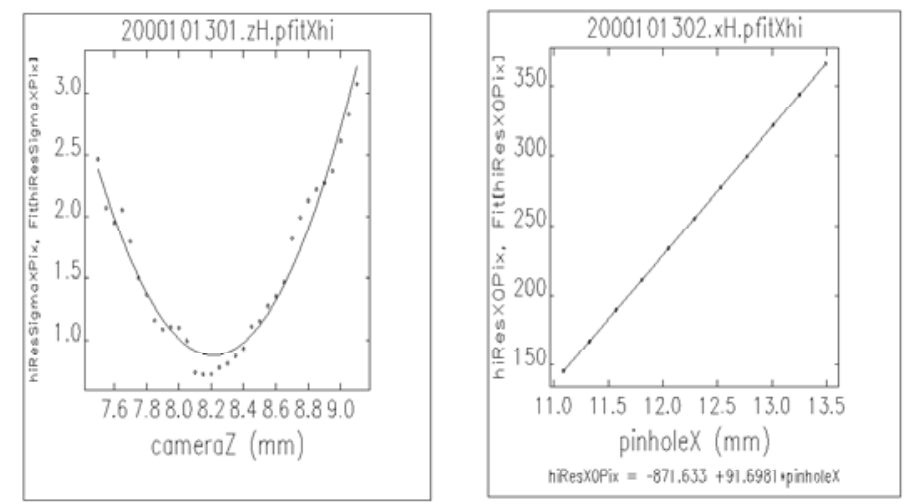

FIGURE 10. (Left) Longitudinal scans of pinhole target pinpoints the focus distance and resolution degradation due to defocusing. (Right) Transverse scans are used to calibrate the camera pixel size.

To summarize the exercise in this section, we make the following observations about typical beam flags with $\sigma_{0} \geq 30 \mu \mathrm{m}$ and WD $\leq 300 \mathrm{~mm}$.

(1) Requirements for the imaging optics are well within the current technological capabilities. Industrial-grade optical components are sufficient for the application.

(2) To consistently obtain 5\% or better measurement accuracy, due attention should be paid to reproducible focus, reliable calibration, mechanical stability, and overall serviceability.

(3) Current crystal scintillators have high conversion efficiency, but problematic spatial resolution (blurring); while the OTR screens have good spatial resolution but low conversion efficiency. Major advances in efficient, high-resolution converter screens are highly desirable.

\section{SYNCHROTRON RADIATION IMAGING SYSTEM}

Synchrotron radiation (SR) is produced when high-energy charged particles pass a magnetic field and their trajectory is bent on a circular orbit $[17,18]$. The wavelength of SR spans from infrared to hard x-rays. Its use for diagnostics offer the following advantages: 
(1) The diagnostic is not intrusive. Particle beams can be studied without disturbing accelerator operation.

(2) The radiation process is fast. At short wavelength, the photon pulse generated by a single electron lasts only $\sim \rho / \gamma^{3} c$ ( $\rho=$ electron trajectory radius), usually lasting less than $1 \mathrm{fs}$. At the long wavelength limit, the pulse length is determined by the optics / spectrometer and could last several wavelengths. Hence the particle bench length can be measured and longitudinal dynamics can be studied in time-domain with streak cameras.

We will discuss the optical synchrotron radiation (OSR) and $\mathrm{x}$-ray synchrotron radiation (XSR) separately due to the substantial differences in their instrumentation.

\section{Optical Synchrotron Radiation Imaging}

For most rings, the OSR wavelength is longer than the critical wavelength $\left(\lambda_{c}=4 \pi \rho / 3 \gamma^{3}\right)$, and it is directed in the forward direction over a cone with the opening angle,

$$
\sigma_{y}^{\prime}=0.73\left(\frac{\lambda}{\rho}\right)^{1 / 3}, \quad\left(\lambda>\lambda_{c}\right) .
$$

For optical wavelength ( $\lambda \square 0.1 \mathrm{~nm}$ ), this angle is in the range of 1 to $3.4 \mathrm{mrad}$ over a wide energy range, since $\rho$ is in the range of $1-5 \mathrm{~m}$ for low-energy rings $(\gamma<2000)$ and $10 \mathrm{~m}-40 \mathrm{~m}$ for high-energy rings $(\gamma>2000)$. We can also derive the diffractionlimited resolution

$$
\sigma_{y}=\frac{\lambda}{2 \sigma_{y}^{\prime}}=0.68\left(\rho \hbar^{2}\right)^{1 / 3} .
$$

For $\lambda=630 \mathrm{~nm}$, we have $\sigma_{y}[\mu \mathrm{m}]=15 \rho[\mathrm{m}]^{1 / 3} \square 15-50$. At high-energy rings, where the beam is smaller and the limit is higher, this constraint is very important. Much ingenuity has gone into overcoming the engineering challenges and realizing the diffraction limit.

(1) Acceleration chamber modification: To realize the full resolution, vertical and horizontal acceptance of the imaging system needs to be greater than $6 \cdot \sigma_{y}^{\prime}$, often in the range of $10-30 \mathrm{mrad}$. Such a large opening is difficult to obtain since it conflicts with machine designers' desire of minimizing chamber discontinuities and wakefield generation.

(2) High angular resolution: The large particle orbit radius results in long working distance. Angular sizes of the beam often fall below $10 \mu \mathrm{rad}$. Mechanical supports of the optical elements need to be very sturdy. If a high flux throughput is desired, or temporal dispersion is of concern (for example, in a high-speed imaging system), an all-mirror imaging system is recommended.

(3) Air current: If a long transport is used, air current and density fluctuation in the air could easily deflect the light beam by microradians. An enclosed or evacuated beam path is recommended. 
(4) Cooling of the first mirror: The synchrotron radiation power could range from several watts to kilowatts. A mirror heated in the front surface distorts significantly. A uniform heating of the surface would bend the mirror and move the focus in the $y$-plane (bending plane) downstream from that in the $x$-plane. The nonuniform heating, due to the concentrated x-ray fan on the orbit plane, creates a high-stress/strain region. It pushes the upper and lower portion of the mirror to rotate in opposite directions (Fig. 11A) and results in a vertically split image. To mitigate the problem, several approaches have been tried with partial success, all using the fact that the opening angle of the visible light is much larger than that of x-rays: half of the mirror outside of the x-ray fan [19], a grazing incidence mirror to spread out the heat load [20], an upstream blocking tube to block the x-rays, and a mirror with a slot aperture to allow the x-ray fan to pass [21].

One of the important features of the OSR imaging is its time-resolved imaging capability. Figure 11B shows sample streak camera pictures using OSR.

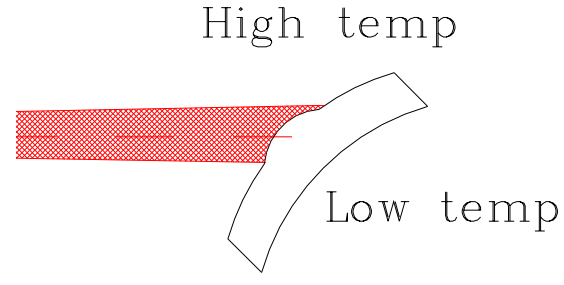

(A)

FIGURE 11. (A) Deformation of mirror under synchrotron radiation heating. (B) Streak camera images of a bunch train in the APS storage ring [22].

\section{OSR Interferometer}

Before discussing the OSR interferometer, we revisit the Helmholtz diffraction integral, Eq. (11), in the one-dimensional case. This time, we divide the entrance aperture into slits of width $\Delta$ and group together the integral from pairs of symmetrically placed slits, $n \Delta \leq|\theta| \leq(n+1) \Delta, n=0,1,2, \ldots$. The resulting amplitude from the n-th pair is

$$
A_{\text {focus }}^{(n)}(x) \cdot e^{i \omega t} \square 2 \Delta \cdot A\left[\left(n+\frac{1}{2}\right) \Delta\right] \cdot \cos \left[\frac{x}{\lambda}\left(n+\frac{1}{2}\right) \Delta\right] \frac{\sin \left(\frac{x \Delta}{2 \lambda}\right)}{\frac{x \Delta}{2 \lambda}} \cdot e^{i \omega t} .
$$

We can see that the amplitude is the product of three functions (Fig. 12): the light amplitude at the slits, a cosine function with (spatial) frequency determined by the angular distance of the slits $n \Delta$, and a sinc function determined by the slits' width. For the total sum to form a point-like image, all these functions need to have the same phase at the focal point, $x=0$. When the first mirror distorts symmetrically, a phase shift is added to the time-dependent factor, $e^{i(\omega t+\phi(n \Delta))}$, and when it distorts asymmetrically, another factor is added to the spatial oscillating factor, 
$\cos \left[\frac{x}{\lambda}\left(n+\frac{1}{2}\right) \Delta+\psi(n \Delta)\right]$. These additional phase shifts make it impossible to obtain a sharp, single-peaked PSF. This problem has an analogy in electronics: To faithfully amplify a short pulse, an amplifier needs to have a flat gain curve in a wide frequency band and a linear phase shift as a function of frequency, with good signal-to-noise ratio. When these conditions cannot be met, electronics engineers often use narrow band electronics to extract partial information from the pulse. Information about the shape of the pulse, or existence of side pulses is often lost in such analyses. Similarly, the OSR interferometer tries to overcome the mirror distortion by analyzing the information from one pair of slits at a time. Due to the transverse size of the source, the observed interference fringes are the convolution of the source distribution with the single particle diffraction pattern. As a result, the blur of the interference fringes are different for different spatial frequencies, hence the source size can be obtained through analyses of the fringe visibilities as a function of spatial frequency $[4,23]$.

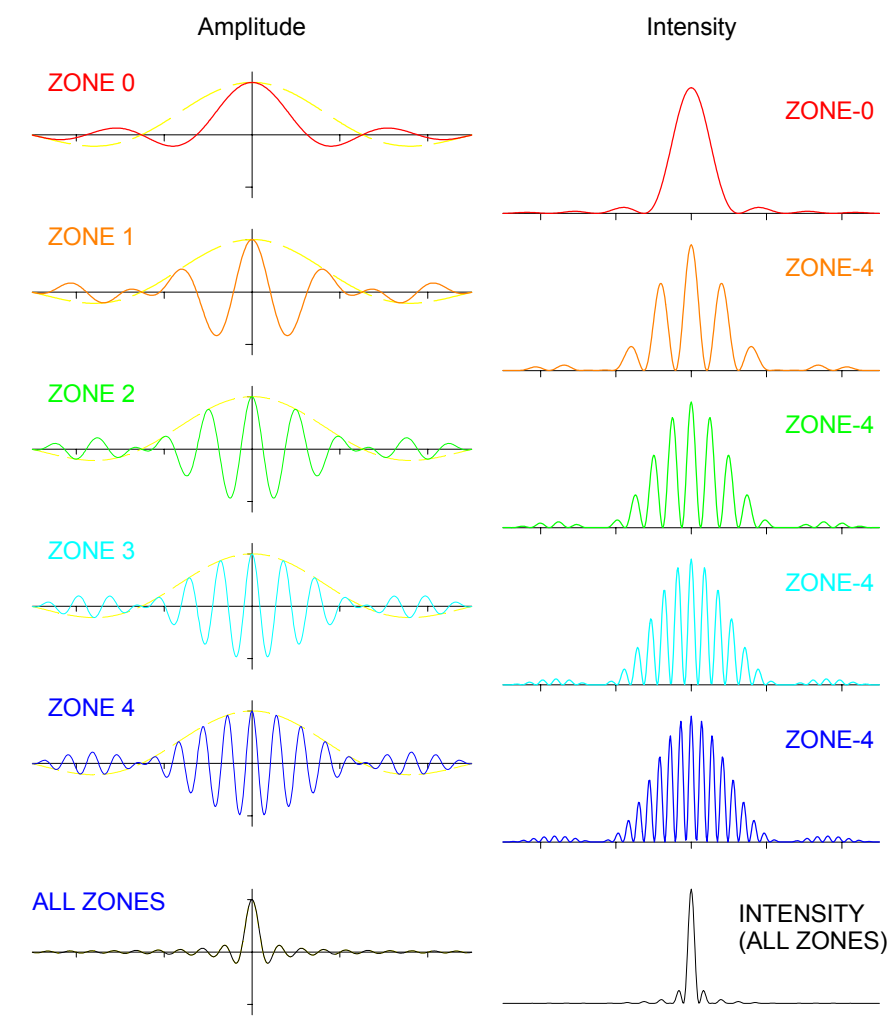

FIGURE 12. (Left) light amplitudes from symmetric slits pairs (zones) at increasing distance from the optical axis. The bottom figure shows the sum of all amplitudes, showing formation of the PSF. (Right) Light intensity at focal plane if only one zone is open. The bottom figure shows the intensity when all zones are open.

The OSR interferometer is fairly effective in overcoming the mirror distortion problem. It has rapidly developed into an important technique for beam size measurements in the past several years. 


\section{X-ray Synchrotron Radiation Imaging}

For most rings, $\mathrm{x}$-ray synchrotron radiation (XSR) wavelength is near or shorter than the critical wavelength. Its opening angle is smaller than that of the OSR

or in practical units

$$
\sigma_{y^{\prime}} \approx \frac{0.64}{\gamma} \sqrt{\frac{\lambda}{\lambda_{c}}}, \quad\left(\lambda<\lambda_{c}\right)
$$

$$
\sigma_{y^{\prime}}[\mu \mathrm{rad}] \cong 190 \sqrt{\lambda[A] B[T]}, \quad\left(\lambda<\lambda_{c}\right),
$$

where $\mathrm{B}$ is the bend magnet field. If the full wavefront is used, the diffraction-limited resolution is

$$
\sigma_{R}[\mu m] \square \frac{\lambda}{2 \sigma_{y^{\prime}}}=0.26 \sqrt{\frac{\lambda[A]}{B[T]}}, \quad\left(\lambda<\lambda_{c}\right) .
$$

For x-ray wavelength $(\lambda \square 0.1 \dot{A})$, the rms opening angle is about $50 \mu \mathrm{rad}$ and diffraction-limited resolution is less than $0.1 \mu \mathrm{m}$. To the author's knowledge, no one has been able to obtain such resolution for direct imaging, most likely due to the difficulty of fabricating atomic-accuracy $x$-ray optics. While focusing optics have been used to directly image the beam, including Kirkpatrick-Beaz mirrors and zone plates [24], it is the pinhole camera that enjoys the most popularity $[25,26]$.

\section{X-ray Pinhole Camera}

The resolution of the pinhole camera has been discussed in a number of works. One approach is to calculate the geometric shadow and Fraunhofer diffraction separately and take the convolution of the two as the true resolution of the pinhole camera (mixed model). Another is to use a Fresnel diffraction approximation. Their results are summarized in Fig. 13. The width of the slits is in units of $\sqrt{\lambda f}$, while the resolution at the object plane is in units of $S^{\prime} \sqrt{\lambda / f}$, where

$$
\frac{1}{f}=\frac{1}{S}+\frac{1}{S^{\prime}},
$$

and $\mathrm{S}$ and $\mathrm{S}^{\prime}$ are the distance of the source and detector from the pinhole aperture, respectively. Note that the optimum aperture in Fresnel model is about 50\% larger than that of the mixed model, and the optimum resolution is about $30 \%$ better. This can be understood in terms of the Fresnel zones: when the aperture is just slightly larger than the first Fresnel zone, all light amplitudes from the aperture are of the same sign at the image plane. Hence the central peak is the highest, and by energy conservation, the peak is also narrowest at the same time.

Figure 14 shows the pinhole camera used in the APS storage ring. The first component is a $1-\mathrm{mm}$ water-cooled aperture used to restrict the radiation power load of the pinhole aperture to several watts. The pinhole apertures are located $9 \mathrm{~m}$ from the source. They are made of four independent tungsten blades with openings usually set at $15 \mu \mathrm{m}$. Temperature-regulated water flow is used to maintain the blade at a 
constant temperature to avoid current dependence of measurements. The vacuum window is located at $16 \mathrm{~m}$, close to the detector to minimize the effect of small-angle scattering. A scintillator / optics / camera combination reads out the x-ray image.

\section{EFFECTIVE RESOLUTION IN MIXED MODEL}

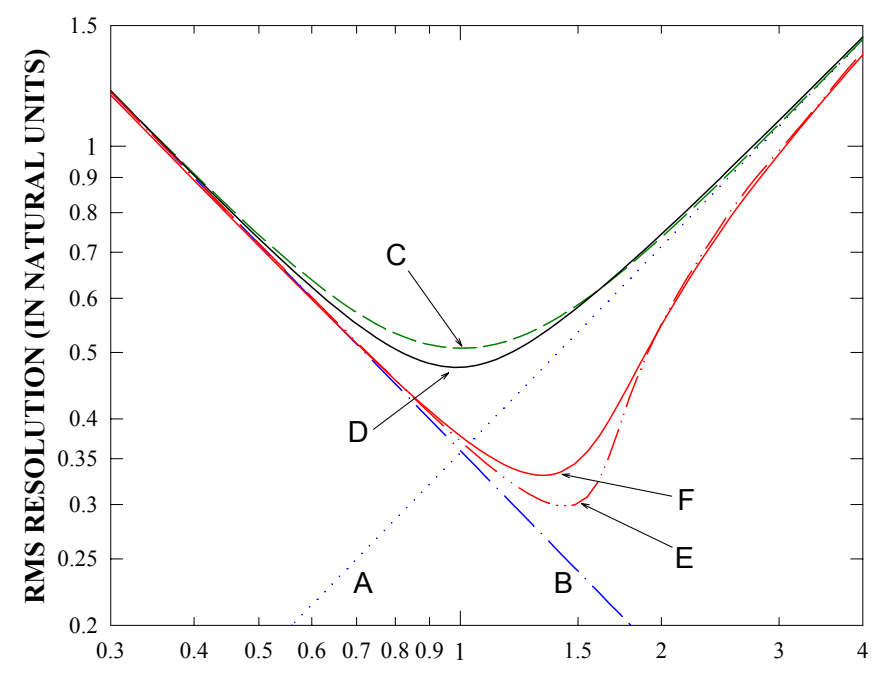

APERTURE WIDTH (IN NATURAL UNITS)

FIGURE 13. Effective resolution of pinhole cameras with different models. (A) Geometrical shadow; (B) Fraunhofer diffraction peak; (C) quadrature sum of the widths of geometric and Fraunhofer peaks; (D) convolution of geometric shadow and the Fraunhofer diffraction peak; (E) monochromatic Fresnel diffraction peak; and (F) multiwavelength Fresnel diffraction with rms spectral width equal to $30 \%$ of the center wavelength. The natural units of the aperture and the source are defined as $\sqrt{\lambda f}$ and $S^{\prime} \sqrt{\lambda / f}$, respectively.

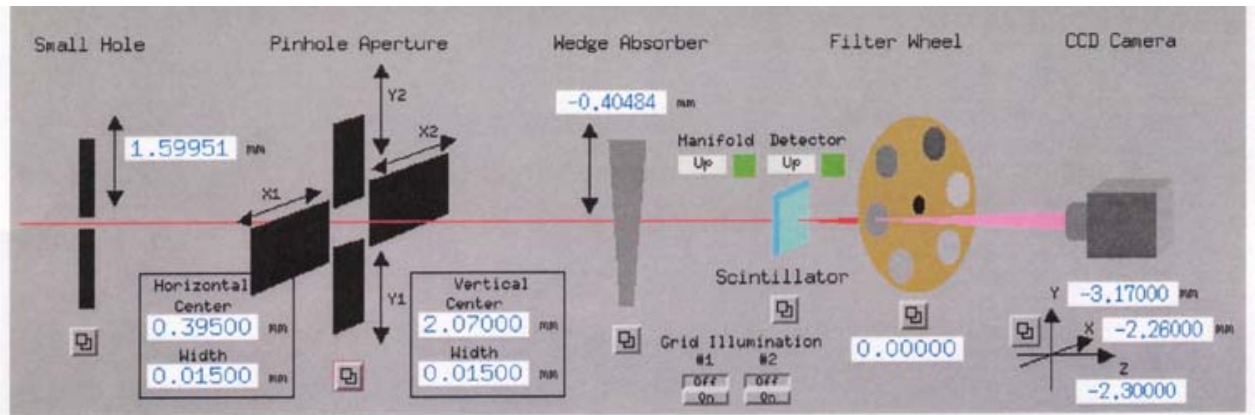

FIGURE 14. Control screen schematic of the APS storage ring pinhole camera.

\section{ACKNOWLEDGMENTS}

The author wishes to thank A. Lumpkin, G. Decker, and O. Singh for their encouragement and support. This work is supported by the U. S. Department of Energy, Office of Basic Energy Sciences, under contract no. W-31-109-ENG-38. 


\section{REFERENCES}

1. Wilke, M., "Optical and X-ray Imaging of Electron Beam Using Synchrotron Emission," BIW94, AIP Proc. 333, 1994, pp. 128-147.

2. Hofmann, A., "Beam Diagnostics and Applications," BIW98, AIP Conf. Proc. 451, 1998, pp. 3-22.

3. Jung, R., "Image Sensor Technology for Beam Instrumentation," BIW98, AIP Conf. Proc. 451, 1998, pp. 74-93.

4. Mitsuhashi , T., "Spatial Coherency of The Synchrotron Radiation at the Visible Light Region and its Application for the Electron Beam Profile Measurement," Proc. Particle Accelerator Conference 1997, 1998, pp. 766-768.

5. Heimann, P. et al., "Experimental Characterization of ALS Undulator Radiation," Rev. Sci. Instrum. 66, 1885 (1995).

6. Yang, B. et al., "Design and Performance of a Compact Imaging System for the APS Linac Bunch Compressor," Proc. Particle Accelerator Conference 2001, 2001, pp. 2335-2337.

7. Borland, M et al., "A Highly Flexible Bunch Compressor for the APS LEUTL FEL," Proc. LINAC 2000, 2000, pp. 863-865.

8. Autrata, R. et al., "Single Crystal Aluminates: A New Generation of Scintillators," Scanning Electron Microscopy II, 1983, pp. 489-500.

9. Graves, W. S. and Johnson, E.D., "A High Resolution Electron Beam Profile Monitor," Proc. Particle Accelerator Conference 1997, 1998, pp. 1993-1995.

10. Rodnyi, P. A., Physical Processes in Inorganic Scintillators, New York, CRC, 1997.

11. Lumpkin A. H. et al., "Time Resolved Imaging for the APS Linac Beams," Proc. of LINAC 98, 1998, pp. 529-531.

12. Murokh, A. et al., "Limitations on Measuring a Transverse Profile of Ultra-Dense Electron Beams with Scintillators," Proc. Particle Accelerator Conference 2001, pp. 1333-1335.

13. Fiorito, R. B. and Rule, D. W., "Optical Transition Radiation Beam Emittance Diagnostics", AIP Conference Proceedings No. 319, 1934, pp. 21-37.

14. Born, M. and Wolf, E., Principles of Optics, Oxford, Pergamon, 1980.

15. Artru X. et al., "Resolution Power of Optical Transition Radiation: Theoretical Considerations," Nucl. Inst. Meth. B145, 160 (1998).

16. ZEMAX Optical Design Program, Focus Software, Tucson, 1992-2002.

17. Kim, K. J., "Characteristics of Synchrotron radiation," AIP Conf. Proc. 184, 1989, pp. 567-632.

18. Koch, E.-E. ed., Handbook on Synchrotron Radiation, North-Holland, Amsterdam, 1983.

19. Scheidt, K., "UV and Visible Light Diagnostics for The ESRF Storage Ring," Proc. European Particle Accelerator Conference 1996, 1996, pp. 1621-1623.

20. Fisher, A. S. et al., "Design and Initial Commissioning of Beam Diagnostics for the PEP-II B Factory," Proc. Particle Accelerator Conference 1997, 1998, pp. 2253-2255.

21. Rotela, E. R. et al., " High Precision, High Heat-Load Mirror for the APS Diagnostics Beamline," Proc. SRI95, 1995, CD-ROM.

22. Yang, B. et al., "Characterizing Transverse beam Dynamics at the APS storage Ring Using a DualSweep Streak Camera," AIP Conf. Proc. 451, 1998, pp. 224-236.

23. Hiramatsu, S. et al., "Measurement of Small Beam Size by the Use of SR Interferometer," Proc. Particle Accelerator Conference 1999, 1999, pp. 492-494.

24. Keller R. et al., "Electron Beam Diagnostics Using Synchrotron Radiation at the Advanced Light Source," AIP Conf. Proc. 390, 1996, pp. 240-247.

25. Yang, B. et al., "Recent Developments in Measurement and Tracking of the APS Storage Ring Beam Emittance," AIP Conf. Proc. 546, 2000, pp. 622-630.

26. Limborg, C. et al., “A Pinhole Camera for SPEAR 2,” Proceedings of EPAC 2000, 2000, pp. 17741776 . 